\title{
Propriedades psicométricas da versão brasileira do Physical Edu- cation Teaching Processes Questionnaire
}

\section{Psychometric properties of the Brazilian version of the Physical Education Teaching Processes Questionnaire}

Marcos Gimenes Fernandes ${ }^{1 *}$, Sandra Adriana Neves Nunes ${ }^{2}$, Gidafle Gama Silva ${ }^{1}$, Helder Miguel Fernandes 3,4

ARTIGO ORIGINAL | ORIGINAL ARTICLE

\begin{abstract}
O presente estudo teve como objetivos: i) traduzir e adaptar para o contexto brasileiro (equivalência semântica/conceitual e validade de conteúdo) o Physical Education Teaching Processes Questionnaire; e ii) verificar as suas propriedades psicométricas (confiabilidade e validade fatorial). A amostra foi composta por 301 alunos (140 do sexo masculino e 161 do sexo feminino) de dua escolas públicas da Bahia, com idades compreendidas entre 12 e 18 anos $(M=16.35 ; D P=1.73)$. A consistência interna e validade fatorial do instrumento foram analisadas através do alpha de Cronbach e análise fatorial confirmatória (AFC), respectivamente. A confiabilidade dos fatores variou entre .54 e .78. Os resultados da AFC indicaram que a versão original (20 itens e 6 fatores) do Physical Education Teaching Processes Questionnaire revelou uma boa adequação estrutural. Estes resultados sugerem que a versão brasileira deste instrumento possui propriedades psicométricas adequadas para ser utilizado no contexto escolar brasileiro.
\end{abstract}

Palavras-chave: validade; confiabilidade; questionário.

ABSTRACT

The present study aimed to: i) translate and adapt to the Brazilian context (semantic equivalence /conceptual and content validity) the Physical Education Teaching Processes Questionnaire; and ii) examine its psychometric properties (factorial validity and fidelity). The sample consisted of 301 students (140 males and 161 females) of two public schools of Bahia, aged between 12 and 18 years $(\mathrm{M}=16.35$, SD $=1.73)$. The internal consistency and factorial validity of the instrument were analyzed using Cronbach's alpha and confirmatory factor analysis (CFA), respectively. The reliability of the factors varied between .54 and .78 . The results of the CFA indicated that the original version (20 items and 6 factors) Physical Education Teaching Processes Questionnaire showed good structural adequacy. These results suggest that the Brazilian version of the instrument has adequate psychometric properties for use in the Brazilian school context

Keywords : validity, reliability, questionnaire.

\footnotetext{
Artigo recebido a 29-04-2014; Aceite a 10-06-2014

${ }^{1}$ Universidade Estadual de Santa Cruz, Brasil

2 Universidade Federal do Sul da Bahia, Brasil

${ }^{3}$ Centro de Investigação em Desporto, Saúde e Desenvolvimento Humano (CIDESD), Portugal

${ }^{4}$ Universidade de Trás-os-Montes e Alto Douro, Vila Real, Portugal

* Autor correspondente: Campus Soane Nazaré de Andrade - Rod. Jorge Amado, km 16 - Salobrinho, Ilhéus BA, 45662-900, Brasil, E-mail: gimenes@uesc.br
} 


\section{INTRODUÇÃO}

Nos últimos anos, tem sido crescente o número de estudos (Hashim, Grove, \& Whipp, 2008; Motl et al., 2001; Yli-Piipari, Watt, Jaakkola, Liukkonen, \& Nurmi, 2009) que objetivam entender o prazer/divertimento (enjoyment) no contexto da educação física escolar. De acordo com Scanlan e Simons (1992), esta dimensão psicológica refere-se a uma resposta afetiva e positiva com relação a esportes que reflete sentimentos de prazer e diversão.

Segundo Shephard e Trudeau (2000), há evidências empíricas da relação entre o prazer/diversão na prática da educação física escolar com a satisfação da prática e sua manutenção de atividade física na vida adulta. Ou seja, as evidências indicam que adolescentes que experimentam prazer e/ou diversão pelas aulas de educação física escolar tendem a se tornar adultos que também encontram maior satisfação nos esportes e/ou na atividade física.

Com base no que foi exposto anteriormente, entende-se que é de significativa importância o papel da educação física escolar no estabelecimento de hábitos saudáveis entre crianças e adolescentes, haja vista que tais hábitos parecem repercutir positivamente na vida adulta. De fato, segundo Bankof e Zamai (2012) a educação física escolar está diretamente relacionada ao desenvolvimento motor e a aptidão física para o bem estar e a saúde dos indivíduos. Nesse sentido, estudos que se propõem a investigar as propriedades psicométricas dos instrumentos que medem a satisfação nas aulas de educação física em crianças e adolescentes são de fundamental importância, uma vez que, ao serem validados, podem ser utilizados por profissionais da área para subsidiar estratégias de intervenção, que visem a despertar na criança o hábito saudável da prática de atividade física.

Como em toda atividade humana, há fatores psicológicos envolvidos no hábito saudável da prática de atividade física. Dentre os fatores psicológicos que permeiam a participação de adolescentes na prática regular de atividade física, a motivação tem recebido atenção especial (Marcus \& Sallis, 1997; Morgan et al., 2003;
Pate, 1997). Uma das teorias da motivação que mais tem sido testada empiricamente no contexto esportivo e educacional é a Harter Competence Motivation Theory (Feltz \& Petlichkoff, 1983; Klint \& Weiss, 1987). O aspecto básico dessa teoria é a percepção da competência, a qual aumenta a motivação intrínseca e o desejo de permanecer envolvido com grande esforço numa atividade (Harter, 1981). Nessa mesma perspectiva, a Cognitive Evaluation Theory, integrada na macro teoria da autodeterminação (Deci \& Ryan, 1985), defende que a motivação varia de acordo com as mudanças da percepção da competência, ou seja, a motivação intrínseca aumenta com uma percepção positiva de sua própria habilidade, enquanto que uma percepção negativa de competência parece estar associada a uma diminuição da motivação intrínseca.

Segundo Duda (1992) e Nicholls (1989), o indivíduo pode demonstrar sua competência através de dois padrões de orientação motivacional. Um padrão denominado de orientação à tarefa, o qual se caracteriza por percepção da competência relacionada ao desenvolvimento pessoal e autorreferenciada e, o outro padrão, chamado de orientação ao ego, a qual a percepção da competência se caracteriza por uma preocupação com o desempenho e uma comparação com outros indivíduos.

De acordo com Carroll e Loumidis (2001) e Cairney et al. (2012), a percepção de competência está diretamente relacionada ao prazer com a Educação Física Escolar, ou seja, crianças com altos níveis de percepção de competência na área esportiva tendem a estar satisfeitas com a educação física. Para além disso, Carroll e Loumidis (2001) relatam que adolescentes com alta percepção de competência, participam mais frequentemente de atividades físicas fora da escola. Além disso, parece haver diferenças de gênero, uma vez que meninos têm maior participação em atividades físicas, têm maior percepção de competência e maior satisfação nessas atividades quando comparados às meninas.

Conforme Wankel (1993), o prazer é um fator crítico e determinante para a motivação e participação contínua na prática esportiva. 
Weiss e Petlichkoff (1989) advertem que crianças são mais inclinadas a participarem de atividade física quando têm experiências de satisfação e as chances de continuarem na atividade aumentam de acordo com sua motivação intrínseca. Segundo Scanlan e Lewthwaite (1986) o prazer está positivamente correlacionado com a vontade de participação.

Alguns estudos foram conduzidos para investigar os fatores que interferem no prazer e divertimento nas aulas de educação física. Por exemplo, Dishman et al. (2005) propõe algumas estratégias instrucionais com o objetivo de aumentar a satisfação dos alunos com as aulas de educação física e, consequentemente, os níveis de atividade física, a saber: a) atividades diferenciadas para meninos e meninas, b) escolha de atividades de ampla variedade, c) atividade preferidas pelos alunos, d) minimizar a competição, e) atividades em pequenos grupos. Wilkinson e Schneck (2003), por sua vez, concluem que pais bem informados sobre a importância da educação física escolar, tendem a se envolver mais com as aulas de educação física dos filhos, o que, pode desencadear nos filhos um maior prazer nas aulas de educação física escolar. Há também evidencias de que o prazer na educação física esteja associada à satisfação com outros agentes sociais, como os pares (Carlson \& Hastie, 1997) ou professores (Cecchini et al., 2001).

Inspirados nos estudos citados anteriormente, Hashim et al. (2008) propõem o Modelo de Prazer em Educação Física e Atividade Física, baseados num modelo teórico de enjoyment (Scanlan, Carpenter, Lobel, \& Simons, 1983), que investiga seis processos ou dimensões que podem gerar nos alunos satisfação nas aulas de educação física. Os autores, a partir desse modelo, desenvolveram um instrumento psicométrico (Physical Education Teaching Processes Questionnaire) que incorpora essas seis dimensões relacionadas ao prazer dos alunos nas aulas de educação física. Estes autores recorreram a procedimentos de Análise Fatorial Exploratória e Análise Fatorial Confirmatória para desenvolver um questionário de vinte indicadores (questões do teste) que verificam essas seis variáveis latentes, a saber: a competência comparada aos pares, a motivação gerada pelo professor, a interação entre pares, o envolvimento dos pais, a competência autorreferenciada e a atividade gerando motivação. O referido instrumento alcançou bons índices de ajustamento (GFI = .90; CFI $=.95$ e RFMSEA $=.60$ ). Dessa forma, os autores recomendam a utilização do Physical Education Teaching Processes Questionnaire para avaliar diversos processos relacionados à satisfação dos alunos com as aulas de educação física escolar.

No nosso conhecimento não há estudos brasileiros de validação desse instrumento, o qual avalia o prazer com a Educação Física escolar em crianças e adolescentes. Diante do exposto, o presente estudo pretende colmatar a lacuna no que diz respeito à falta de um instrumento com a validade de constructo suportada por Análise Fatorial Confirmatória no contexto cultural brasileiro. Dessa forma, o presente estudo teve como objetivos: i) proceder à tradução e adaptação transcultural (equivalência semântica/conceitual e validade de conteúdo) do Physical Education Teaching Processes Questionnaire (Hashim et al., 2008); e ii) verificar as suas propriedades psicométricas (confiabilidade e validade fatorial).

\section{MÉTODO}

\section{Participantes}

A seleção dos participantes foi do tipo não probabilística e intencional, composta por 301 alunos (140 do sexo masculino e 161 do sexo feminino) de duas escolas públicas da Bahia, com idades compreendidas entre 12 e 18 anos $(M=16.35 D P=1.73)$. No que concerne as séries desses alunos, obedeceu a seguinte distribuição: $7^{\mathrm{a}}$ série $(11.5 \%)$, $8^{\mathrm{a}}$ série $(15 \%)$ e $9^{\mathrm{a}}$ série $(12 \%)$ do ensino fundamental, e $1^{\text {a }}$ série $(27$ $\%), 2^{a}$ série $(14.5 \%)$ e $3^{\text {a }}$ série $(20 \%)$ do ensino médio.

\section{Instrumento}

O Physical Education Teaching Processes Questionnaire foi desenvolvido por Hashim et al. (2008) e é constituído por 20 itens distribuídos em seis sub-escalas, as quais verificam processos relacionados à satisfação 
dos alunos nas aulas de educação física escolar, a saber: competência comparada aos pares (itens $3,6,8$ e 13), motivação gerada pelo professor (itens 9,16 e 19), interação entre pares (itens 15 e 17), envolvimento dos pais (itens 1 e 20), competência auto-referenciadas (itens 4, 5, 7 e 14) e atividade gerando motivação $(2,10,11,12$ e 18). Hashim et al. (2008) reportaram índices de confiabilidade superiores ao usual critério $(\alpha$ $>0.70$ ) para todas essas dimensões. As afirmações serão respondidas de acordo com uma escala do tipo Likert de cinco pontos $(1=$ Discordo Completamente; 2 = Discordo; $3=\mathrm{Nem}$ Discordo Nem Concordo; $4=$ Concordo; e $5=$ Concordo Completamente). Os escores da cada fator são somados e divididos pelo número de itens do respectivo fator (sub-escala).

\section{Procedimento}

$O$ processo de tradução e adaptação transcultural do Physical Education Teaching Processes Questionnaire envolveu duas etapas (Fernandes \& Fernandes, 2015), antes da análise das suas propriedades psicométricas.

\section{Tradução e adaptação cultural}

A primeira etapa consistiu na tradução/retrotradução (método de backtranslation) do instrumento (Brislin, 1970). Este procedimento contou com a colaboração de dois psicólogos especialistas bilíngues (português e inglês) (Fernandes, Nunes, Raposo, Fernandes, \& Brustad, 2013; Fernandes, Vasconcelos-Raposo, \& Fernandes, 2012). Num primeiro momento, um especialista fluente em ambas as línguas traduziu o instrumento do inglês para português do Brasil; posteriormente outro especialista traduziu o instrumento do português do Brasil para o inglês sem consultar a versão original. $O$ resultado foram duas versões do instrumento, uma em cada língua. Num segundo momento, os especialistas se reuniram para avaliar e realizar as modificações na versão preliminar. A versão em inglês foi comparada com a versão original, tendo-se verificado uma equivalência semântica e de conteúdo. A versão da língua portuguesa foi revisada em oito itens e correções foram realizadas com relação à especificidade de alguns termos técnicos, de modo a ser assegurado o consenso entre especialistas e não existirem incompatibilidades com a versão original. Esta consulta também serviu para se analisar a forma e conteúdo dos itens em termos de clareza, compreensibilidade e adequação (Alexandre \& Coluci, 2011).

Numa segunda etapa, a avaliação da validade de conteúdo foi verificada por um comitê constituído por cinco doutores em Eucação Fisica com experiência na área de pesquisa. Num primeiro momento, foi solicitado individualmente aos integrantes do comitê que indicassem a sua concordância ou não em relação à inclusão dos itens nos seus respectivos fatores. Num segundo momento foi calculado a percentagem de pertinência e concordância dos itens nos seus respectivos fatores, tendo por base as avaliações do comitê numa escala de 10 pontos ( 1 = nada pertinente/importante a $10=$ extremamente pertinente/importante). Este processo permitiu o cálculo do índice de validade de conteúdo (IVC) (Alexandre \& Coluci, 2011), tendo-se obtido valores médios superiores a $80 \%$ para todos os itens, o que suporta a adequação/inclusão dos itens nos seus respetivos fatores.

\section{Administração do instrumento}

Para a coleta de dados, foi realizado um prévio contato com os diretores das escolas para que pudesse ser obtida a devida autorização e a indicação das salas e dos horários em que a coleta poderia ser feita. Após esta autorização, ocorreu a assinatura de Termo de Consentimento Livre e Esclarecido (TCLE) pelos alunos com 18 anos, e pelos pais ou responsáveis, no caso dos alunos menores. O Physical Education Teaching Processes Questionnaire foi aplicado na sala de aula, de forma coletiva, mediante autorização do professor indicado pela diretoria da escola. A duração da aplicação do instrumento foi de $10 \mathrm{a}$ 20 minutos.

O presente estudo foi aprovado pelo Comitê de Ética em Pesquisa (Protocolo 250.483) da Universidade Estadual de Santa Cruz (UESC), de acordo com a Resolução CNS/MS n. $466 / 2012$. 


\section{Análise estatística}

De início, foi verificada a estatística descritiva das variáveis através da média, desviopadrão, mínimo e máximo. Para verificar a assimetria da distribuição das médias foram utilizados os coeficientes assimetria (skewness) e achatamento (kurtosis), sendo considerados valores aceitáveis para os verificados no intervalo entre $-1.96 \mathrm{e}+1.96$. Posteriormente, foi calculada a consistência interna dos fatores através do alfa de Cronbach. Estas análises foram efetuadas no SPSS 17.0.

A Análise Fatorial Confirmatória (AMOS 6.0) foi utilizada para testar o modelo proposto por Hashim et. al. (2008) do Physical Education Teaching Processes Questionnaire, utilizandose o método de estimação maximuum likelihood e respeitando-se um número mínimo de 10 observações por item (Ding, Velicer, \& Harlow, 1995). Após a especificação e estimação do modelo, a sua adequação foi avaliada por um conjunto de índices de ajustamento/adequação. $\mathrm{O}$ valor de $\chi^{2}$ (Qui-quadrado) indica ajustamento quando o valor não é significativo $(p>0.05)$. No entanto, esse teste é sensível ao tamanho da amostra, ou seja, em amostras numerosas o valor tende a ser significativo, embora o modelo possa estar ajustado aos dados. Em razão desse fato, Jöreskog e Sörbom (1989) sugeriram uma razão do Qui-quadrado pelos graus de liberdade $(d f)$, representado por $\chi^{2} / d f$, pelo que Ullman (2001) sugeriu valores abaixo de 2.0 como aceitáveis. Adicionalmente, foram utilizados os seguintes índices de ajustamento: a) CFI (Comparative Fit Index) e GFI (Goodness Fit Index) podendo os seus valores variar de 0 a 1 , sendo que se o valor se encontrar acima de .90 o modelo apresenta um ajuste adequado (Bentler \& Bonett, 1980), enquanto $\mathrm{Hu}$ e Bentler (1999) sugeriram um ponto de corte de 0.95 como indicativo de um bom ajustamento do modelo; b) RMSEA (Root Mean Square Error of Approximation), em que valores menores que 0.08 indicam uma adequação aceitável (Browne \& Cudek, 1983), embora $\mathrm{Hu}$ e Bentler (1999) tenham mais recentemente sugerido um ponto de corte de .06. Por fim, AIC (Akaike Information Criterion) que indica a parcimônia e simplicidade do modelo através do menor valor entre os modelos testados.

\section{RESULTADOS}

\section{Análise descritiva}

As médias dos valores das respostas dos itens situaram-se entre $2.67 \pm 1.09$ (item 13) e 3.87 \pm 1.04 (item 12). Os valores de normalidade univariada (skewness e kurtosis) tenderam a situar-se num intervalo associado a uma distribuição normal (intervalo entre -1.96 e $+1.96)$.

\section{Confiabilidade}

Foi avaliada a consistência interna das escalas do Physical Education Teaching Processes Questionnaire através do alpha de Cronbach. Para as dimensões originais, os resultados foram os seguintes: envolvimento dos pais (.54); atividade gerando motivação (.78); competência comparada aos pares (.70); competência autorreferenciadas (.74); motivação gerada pelo professor (.69) e interação entre pares (.67).

\section{Analise Fatorial Confirmatória}

O modelo original do Physical Education Teaching Processes Questionnaire, o qual contém 20 itens e seis dimensões, alcançou satisfatórios (GFI) e bons ( $\chi^{2} / d f$, CFI e RMSEA) índices de ajustamento, o que demonstra a sua validade fatorial. Os resultados dos índices são apresentados na Tabela 1.

Tabela1

Índices de Ajustamentos da Analise Fatorial Confirmatória.

\begin{tabular}{ccccccc}
\hline & $\chi^{2}$ & $\chi^{2} / d f$ & CFI & GFI & RMSEA (90\%IC) & AIC \\
\cline { 2 - 7 } Modelo original & 225.67 & 1.46 & .958 & .932 & $.039(.027-.050)$ & 335.67 \\
\hline
\end{tabular}

Notas: $\mathrm{O}$ valor de $\chi^{2}$ é significativo $(p<.01)$ 
A análise dos coeficientes estandardizados das cargas fatoriais do modelo de seis dimensões e 20 itens revelou que o carregamento das variáveis observáveis situouse entre .512 (item 13) e .742 (item 19). A correlação entre as dimensões variou entre .175 $(p<.05)$ para a motivação gerada pelo professor e competência comparada aos pares, e $.831(p<.01)$ para competência autorreferenciada e atividade gerando motivação.

\section{DISCUSSÃO}

O presente estudo teve como objetivo verificar a confiabilidade e validade fatorial (validade de constructo) da versão brasileira do Physical Education Teaching Processes Questionnaire (Hashim et al., 2008). Salvo nossos conhecimentos, este é o primeiro estudo a examinar as propriedades psicométricas (Análise Fatorial Confirmatória) do referido instrumento no contexto brasileiro, o qual possibilitará intervenções e futuras pesquisas com objetivo de desenvolver a prática atividade física entre adolescentes, a partir da educação física escolar. De acordo com Wallhead e Buckworth (2004) há uma relação positiva entre prazer com a educação física escolar e prática de atividade física entre crianças e adolescentes. Além disso, a prática de atividade física na infância e adolescência parece estar associada à prática de atividade física na vida adulta (Shepard \& Trudeau, 2000). Por outro lado, para que a haja promoção de atividade física em crianças e adolescentes é necessário que os alunos encontrem prazer na educação física escolar (Dishman et al., 2005; Fairclough, 2003; Fairclough \& Stratton, 2006).

Com relação à tradução e equivalência entre as versões original e brasileira, observou-se uma satisfatória equivalência. Os resultados da verificação da validade de conteúdo (IVC) relativa ao painel de especialistas superaram o ponto de corte de .80 , de acordo com o recomendado pela literatura (Alexandre \& Coluci, 2011). Dessa forma, a versão brasileira do Physical Education Teaching Processes Questionnaire contém um conjunto de itens que determinam o mesmo conteúdo, relacionado a cada dimensão do instrumento (Rubio, Berg-Weger, Tebb, Lee, \& Rauch, 2003).

No que se refere à confiabilidade, a versão brasileira do Physical Education Teaching Processes Questionnaire demonstrou, para a maioria de suas dimensões, satisfatórios índices de consistência interna, semelhante aos resultados do modelo original (Hashim et al., 2008). Relativamente aos resultados de confiabilidade obtidos para as dimensões "motivação gerada pelo professor" (.69) e "interação entre pares" (.67), Loewenthal, (2001) e Marôco e Garcia-Marques (2006) sugerem que valores de .60 ou superiores são adequados para escalas com um reduzido número de itens por fator, como é o caso dessas dimensões, com três e dois itens, respectivamente. Maior precaução torna-se necessária quanto à dimensão "envolvimento dos pais", cuja baixa confiabilidade parece expressar o escasso número de itens que compõem a dimensão. Importa destacar que no estudo (Hashim et al., 2008) de desenvolvimento do referido instrumento, essa dimensão também apresentou o menor índice de confiabilidade, comparada as outras dimensões. Contudo, e de forma geral, os resultados obtidos sugerem existir uma satisfatória homogeneidade e uniformidade entre as respostas dos sujeitos da amostra para as dimensões analisadas (Fernandes, Vasconcelos-Raposo, \& Fernandes, 2013).

Através dos procedimentos de Análise Fatorial Confirmatória, o modelo original (20 itens e seis fatores) do Physical Education Teaching Processes Questionnaire alcançou aceitáveis e bons índices de ajustamento, corroborando com os achados de Hashim et. al. (2008). Dessa forma, foi suportada a validade de constructo do Physical Education Teaching Processes Questionnaire. De acordo com Pasquali (2009), a validade de constructo é considerada a forma mais fundamental de validade dos instrumentos psicológicos, por constituir a maneira direta de verificar a representação comportamental das variáveis latentes. 
O presente estudo teve como principais limitações o processo de amostragem nãoprobabilístico e o fato de não se ter analisado a invariância métrica (Byrne, 2008) por sexo. Dessa forma sugere-se que em futuros estudos seja examinada a invariância métrica por sexo da versão brasileira do Physical Education Teaching Processes Questionnaire em amostras representativas e probabilísticas, assim como, continuar o processo, com validações adicionais, como por exemplo, validade convergente e de critério.

\section{CONCLUSÕES}

Conclui-se que a versão brasileira do Physical Education Teaching Processes Questionnaire, o qual é constituído de 20 itens e seis dimensões, revelou aceitáveis propriedades psicométricas (confiabilidade e validade fatorial) indicando ser uma medida válida para avaliar diversos processos relacionada ao prazer dos alunos com a educação física escolar. É esperado que os conhecimentos obtidos com esta escala auxilie a construção de estratégias para melhorar as aulas de educação física escolar com o objetivo de desenvolver a prazer e motivação nessas aulas, promovendo assim o hábito saudável da prática regular de atividade física.

\section{Agradecimentos:}

Nada a declarar.

\section{Conflito de Interesses}

Nada a declarar.

Financiamento:

Nada a declarar

\section{REFERÊNCIAS}

Alexandre, N. M. C., \& Coluci, M. Z. O. (2011). Validade de conteúdo nos processos de construção e adaptação de instrumentos de medidas. Ciência \& Saúde Coletiva, 16(7), 3061-3068. http://doi.org/10.1590/S141381232011000800006
Bankoff, A. D. P., \& Zamai, C. A. (2012). Estudo do perfil dos professores do ensino fundamental do Estado de São Paulo sobre o conhecimento do tema atividade física e saúde. Revista Brasileira de Atividade Física \& Saúde, 4(3), 35-42.

Bentler, P. M., \& Bonett, D. G. (1980). Significance tests and goodness of fit in the analysis of covariance structures. Psychological Bulletin, 88(3), 588-606. http://doi.org/10.1037/00332909.88.3.588

Brislin, R. W. (1970). Back-Translation for CrossCultural Research. Journal of Cross-Cultural Psychology, 1(3), 185-216. http://doi.org/10.1177/135910457000100301

Browne, M. W., \& Cudek. (1983). Alternate ways of assessing model fit. Em K. A. Bollen \& J. S. Long (Eds.), Testing Structural Equation Models (pp. 136-162). Newbury Park, CA: SAGE.

Byrne, B. M. (2008). Testing for multigroup equivalence of a measuring instrument: a walk through the process. Psicothema, 20(4), 872882.

Cairney, J., Kwan, M. Y., Velduizen, S., Hay, J., Bray, S. R., \& Faught, B. E. (2012). Gender, perceived competence and the enjoyment of physical education in children: a longitudinal examination. The International Journal of Behavioral Nutrition and Physical Activity, 9, 26. http://doi.org/10.1186/1479-5868-9-26

Carlson, T. B., \& Hastie, P. A. (1997). The student social system within sport education. Journal of Teaching in Physical Education, 16(2), 176195.

Carroll, B., \& Loumidis, J. (2001). Children's Perceived Competence and Enjoyment in Physical Education and Physical Activity Outside School. European Physical Education Review, 7(1), 2443. http://doi.org/10.1177/1356336X010071005

Cecchini, J., González, C., Carmona, Á., Arruza, J., Escartí, A., \& Balagué, G. (2001). The influence of the physical education teacher on intrinsic motivation, self-confidence, anxiety, and preand post-competition mood states. European Journal of Sport Science, 1(4), 1-11. http://doi.org/10.1080/17461390100071407

Deci, E. L., \& Ryan, R. M. (1985). Intrinsic Motivation and Self-Determination in Human Behavior. New York: Plenum.

Ding, L., Velicer, W. F., \& Harlow, L. L. (1995). Effects of estimation methods, number of indicators per factor, and improper solutions on structural equation modeling fit indices. Structural Equation Modeling: A Multidisciplinary Journal, 2(2), 119-143. http://doi.org/10.1080/10705519509540000

Dishman, R. K., Motl, R. W., Saunders, R., Felton, G., Ward, D. S., Dowda, M., \& Pate, R. R. (2005). Enjoyment mediates effects of a schoolbased physical-activity intervention. Medicine and Science in Sports and Exercise, 37(3), 478487. 
Duda, J. L. (1992). Motivation in sport settings: A goal perspectives approach. Em G. C. Roberts (Ed.), Motivation in Sport and Exercise (pp. 5791). Champagn, IL: Human Kinetics.

Fairclough, S. (2003). Physical activity, perceived competence, and enjoyment during secondary school physical education. European Journal of Physical Education, 8(1), 5-18.

Fairclough, S., \& Stratton, G. (2006). Effects of a Physical Education Intervention to Improve Student Activity Levels. Physical Education and Sport Pedagogy, 11(1), 29-44.

Feltz, D. L., \& Petlichkoff, L. (1983). Perceived competence among interscholastic sport participants and dropouts. Canadian Journal of Applied Sport Sciences, 8(4), 231-235.

Fernandes, M. G., \& Fernandes, H. M. (2015). Tradução, adaptação transcultural e propriedades psicométricas do TOPS2 em atletas brasileiros. Psicologia: Reflexão e Crítica, 28(1), 136-146. http://doi.org/10.1590/1678-7153.201528115

Fernandes, M. G., Nunes, S. A., Raposo, J. V., Fernandes, H. M., \& Brustad, R. (2013). The CSAI-2: An Examination of the Instrument's Factorial Validity and Reliability of the Intensity, Direction and Frequency Dimensions with Brazilian Athletes. Journal of Applied Sport Psychology, 25(4), 377-391. http://doi.org/10.1080/10413200.2012.74478 0

Fernandes, M. G., Vasconcelos-Raposo, J., \& Fernandes, H. M. (2012). Propriedades psicométricas do CSAI-2 em atletas brasileiros. Psicologia: Reflexão e Crítica, 25(4), 679-687. http://doi.org/10.1590/S010279722012000400007

Fernandes, M. G., Vasconcelos-Raposo, J., \& Fernandes, H. M. (2013). Propriedades psicométricas da versão brasileira do TOPS2. Revista Ibero-Americana de Diagnóstico e Avaliação Psicológica, 36(2), 71-98.

Harter, S. (1981). The development of competence motivation in the mastery of cognitive and physical Skills: Is there still a place for joy? Em C. H. Nadeau (Ed.), Psychology of Motor Behaviour and Sport (pp. 3-29). Champagn, IL: Human Kinetics.

Hashim, H., Grove, J. R., \& Whipp, P. (2008). Validating the youth sport enjoyment construct in high school physical education. Research Quarterly for Exercise and Sport, 79(2), 183-194. http://doi.org/10.1080/02701367.2008.10599 482

Hu, L., \& Bentler, P. M. (1999). Cutoff criteria for fit indexes in covariance structure analysis: Conventional criteria versus new alternatives. Structural Equation Modeling: A Multidisciplinary Journal, 6(1), 1-55. http://doi.org/10.1080/10705519909540118
Jöreskog, K. G., \& Sörbom, D. (1989). Lisrel 7: User's reference guide. Mooresville, IN: Scientific Software.

Klint, K. A., \& Weiss, M. R. (1987). Perceived competence and motives for participation in youth sports: A test of Harter's competence motivation theory. Journal of Sport Psychology, 9(1), 55-65.

Loewenthal, K. M. (2001). An Introduction to Psychological Tests and Scales (2nd ed.). London: Psychology Press.

Marcus, B. H., \& Sallis, J. F. (1997). Determinants of physical activity behavior and implications for interventions. Em A. S. Leon (Ed.), Physical Activity and Cardiovascular Health: A National Consensus (pp. 192-201). Champagn, IL: Human Kinetics.

Marôco, J., \& Garcia-Marques, T. (2006). Qual a fiabilidade do alfa de Cronbach? Questões antigas e soluções modernas. Laboratório de Psicologia, 4(1), 65-90.

Morgan, C. F., McKenzie, T. L., Sallis, J. F., Broyles, S. L., Zive, M. M., \& Nader, P. R. (2003). Personal, social, and environmental correlates of physical activity in a bi-ethnic sample of adolescents. Pediatric Exercise Science, 15(3), 288301.

Motl, R. W., Dishman, R. K., Saunders, R., Dowda, M., Felton, G., \& Pate, R. R. (2001). Measuring enjoyment of physical activity in adolescent girls. American Journal of Preventive Medicine, 21(2), 110-117.

Nicholls, J. G. (1989). Conceptions of Ability and Achievement Motivation. Em R. E. Ames \& Ames (Eds.), Research on Motivation in Education: Student Motivation (pp. 39-73). New York: Academic Press.

Pasquali, L. (2009). Psicometria. Revista da Escola de Enfermagem da USP, 43(S1), 992-999. http://doi.org/10.1590/S008062342009000500002

Pate, R. R. (1997). Physical activity in children and adolescents. Em A. S. Leon (Ed.), Physical Activity and Cardiovascular Health: A National Consensus (pp. 210-217). Champagn, IL: Human Kinetics.

Rubio, D. M., Berg-Weger, M., Tebb, S. S., Lee, E. S., \& Rauch, S. (2003). Objectifying content validity: Conducting a content validity study in social work research. Social Work Research, 27(2), 94-104. http://doi.org/10.1093/swr/27.2.94

Scanlan, T. K., Carpenter, P. J., Lobel, M., \& Simons, J. P. (1983). Sources of enjoyment for youth sport athletes. Pediatric exercise science, 5, 275-285.

Scanlan, T. K., \& Lewthwaite, R. (1986). Social psychological aspects of competition for male youth sport participants: IV- Predictors of enjoyment. Journal of Sport Psychology, 8(1), 125-35.

Scanlan, T. K., \& Simons, J. P. (1992). The construct of sport enjoyment. Em G. C. Roberts (Ed.), 
Motivation in Sport and Exercise (pp. 199215). Champagn, IL: Human Kinetics Books.

Shepard, R. J., \& Trudeau, F. (2000). The legacy of physical education: Influences on adult lifestyle. Pediatric Exercise Science, 12(1), 34-50.

Ullman, J. B. (2001). Structural equation modeling. Em B. G. Tabachnick \& L. S. Fidell (Eds.), Using Multivariate Statistics (4th ed., pp. 653-771). Needham Heights, MA: Allyn \& Bacon.

Wallhead, T. L., \& Buckworth, J. (2004). The Role of Physical Education in the Promotion of Youth Physical Activity. Quest, 56(3), 285-301.

Wankel, L. M. (1993). The importance of enjoyment to adherence and psychological benefits from physical activity. International Journal of Sport Psychology, 24(2), 151-169.
Weiss, M. R., \& Petlichkoff, L. M. (1989). Children's motivation for participation in and withdrawal from sport: Identifying the missing links. Pediatric Exercise Science, 1(3), 195-211.

Wilkinson, C., \& Schneck, H. (2003). The effects of a school physical education and health web site on parental knowledge of the program. The Physical Educator, 60(3), 162-168.

Yli-Piipari, S., Watt, A., Jaakkola, T., Liukkonen, J., \& Nurmi, J.-E. (2009). Relationships Between Physical Education Students' Motivational Profiles, Enjoyment, State Anxiety, and Self-Reported Physical Activity. Journal of Sports Science \& Medicine, 8(3), 327-336. 